Saudi Journal of Biomedical Research

Abbreviated Key Title: Saudi J Biomed Res ISSN 2518-3214 (Print) |ISSN 2518-3222 (Online)

\title{
Effect of Human Papilloma Virus Type 16 and 18 infections on Young Married Women Aged 18-24 Years in River Nile State 2017
}

\section{Abd alwahab Jahalnabi Jubara Bakheet ${ }^{1^{*}}$, Mosab Nouraldein Mohammed Hamad ${ }^{2}$ and Mohammed Ahmed Ibrahim} Holie $^{1}$

${ }^{1}$ Microbiology Department, Faculty of medical laboratory sciences, Alzaiem ALazhari University

${ }^{2}$ Medical Laboratory Sciences Department, Faculty of Health Sciences, Elsheikh Abdallah Elbadri University

DOI: $10.36348 /$ sjbr.2020.v05i01.001

| Received: 28.12.2019 | Accepted: 06.01.2020 | Published: 14.01.2020

*Corresponding author: Abd alwahab Jahalnabi Jubara Bakheet

\section{Abstract}

The study was done in river Nile state, from September to November 2017. The research applied on 50 urine samples which collected from married ladies with young ages (18-24 years) unvaccinated against Human Papilloma Virus 16 and 18 that known as high risk viruses to detect DNA of it and probability of it to cause cervical cancer. The study aimed to detect affection of high risk HPV type 16 or 18 on young married ladies (16-18 years)an vaccinated against it Then probability to cause cervical cancer. Used molecular method to detect presence of high risk HPV DNA (16or 18) in urine sample which collected from the study group. All 50 samples were negative, that mean no detection for HPV16 or 18 DNA.

Keywords: Human Papilloma virus, prevalence, women, Sudan.

Copyright @ 2020: This is an open-access article distributed under the terms of the Creative Commons Attribution license which permits unrestricted use, distribution, and reproduction in any medium for non-commercial use (NonCommercial, or CC-BY-NC) provided the original author and source are credited.

\section{INTRODUCTION}

Cases of cervical cancer were usually associated with Human Papilloma Virus (HPV) infection with two types, HPV16 and HPV18 which were present in $70 \%$ of cases.

HPV types are the most malignant strain, present in 41 to 54\% of all cervical cancers [1] and in many cases (vaginal, vulval, penile and anal cancers) [1].

In 2012 about 528,000new cases and 266,000 deaths from cervical cancer occurred worldwide [2]. About $85 \%$ of these occurred in the developing world. Most HPV infection of the cervix are cleared rapidly by the immune system and do not progress to cervical cancer [2].

Because the process to transforming normal cervical cells into cancerous ones is slow, cancer occurs in people having been infected with HPV for a long time, usually over a decade or more [3].

The normal cervix has two distinct epithelial zones: the ectocervix covered by squamous epithelium, and the endocervix lined by simple glandular epithelium [4]. As a result of metaplasia during adolescence and early adult life the endocervix epithelium is replaced by immature squamous epithelium which eventually matures. This squamous Meta plastic area is called the transformation zone and is by far the most common site for development of cervical cancer. What makes the transformation zone uniquely sensitive to HPV- induced carcinogenesis is not well understood. It may be a consequence of exposure of actively proliferating stem cell or transit amplifying cells in the basal layer to HPV, specific molecular properties of the immature metaplastic epithelial cells or the local immune system.

Underneath the epithelium is a layer of connective tissue and beneath this smooth muscle. There is a local cervical tissue, composed of $\mathrm{T}$ and $\mathrm{B}$ lymphocytes, plasma cells NK cells and macrophages and specialized antigen - presenting cells - langerhans' cells. The local immune system is particularly prominent in certain diseases - for example, lymphoid follicles in follicular cervicitis associated with Chlamydia infection [2].

Carcinoma -in -situ [CIS] has long been recognized as the immediate precursor of invasive cancer, shows a squamous differentiation. The cervical entra epithelial neoplasia [SIN] classification system 
was developed to embrace all potential squamous precancerous lesions and to classify the lesions into grades CIN 1, CIN 2, and CIN 3.

The system is descriptive, the morphological changes are regarded as a continuum and the three grades are defined by arbitrary criteria, the most important of which is the proportion of the epithelium occupied by undifferentiated, basal type cell [3].

CIN was grouped as CIN 1 and CIN 2 respectively. The classification predates and takes no direct account of the oncogenic role or cytopathic effects of different types of HPV.

The characteristic used to define CIN 1 are firstly a degree of epithelial hyperplasia, seen as an increase in thickness of the basal and parabasal layers

All these koilocytosis and multinucleate cells and para keratosis is not specific feature for HPV infection. The diagnosis of CIN 2 is important clinically as it may be transforming lesion and the threshold for treatment in most cervical cancer screening.

CIN 3 is considered the HPV -related immediate intra epithelial precursor of invasive cervical cancer. Papilloma viruses are small.non -enveloped, epithelio-tropic, double stranded DNA virus that infect mucosal and cutaneous epithelia in a wide variety of higher vertebrates in a species. Specific manner and include cellular proliferation. Only bovine papilloma viruses (BPVs) 1and 2 are known to infect mesenchymal tissue and to show cross - species transmission1.2.1.2 Background: More than 100 types of human papilloma viruses have been identified and approximately half of them infect the genital tract. Many types of HPV have been found in cervical cancers. While others are found rarely or not at all in large series of cancers, which gives rise to the nomenclature of ((high -and ((low -risk) HPVs. These other types are associated with other anogenital and oropharyngeal cancers [2].

A number of HPVs have been found to be present in skin cancer in patient who has epidermodysplasia verruciformis (EV), these types are also found in both non -melanoma skin cancers and normal skin [2].

All papilloma viruses share a common genetic structure that is distinct from that of polyoma viruses. A double stranded circular DNA genome encodes approximately eight open reading frames (ORFS).Similarly. All papilloma viruses have non enveloped icosahedral capsid .Understanding of the biology of papilloma virus infection was hidered by the lack of tissue culture systems to propagate the virus ,the lack of animal models for HPVs and difficulties in finding animal models of natural infection. The advent of molecular cloning of HPV genome in the early 1980s provides the first a opportunity to study individual viral genes. However, only in the late 1990s did propagation of viruses in organo typing cultures make the first attempts at viral genetics possible. The availability of complete and partial genomic sequences from a wide variety of HPV types has enabled the establishment of a new taxonomic structure and has provided a window to study the co-evolution of papilloma viruses with their primate hosts. Early evidence suggests that HPV types, as defined by DNA sequencing also remain serological distinct.

Molecular studies now provide a coherent picture of the mechanisms that regulate viral gene expression and replication, nevertheless gaps in the understanding of HPV biology remain Striking progress has been made in defining the activities of viral oncoprotein from high risk genital HPV, in particular HPVs 16 and 18 that promote the disruption of normal cell cycle control [4].

The ability to target the retinoblastoma $(\mathrm{Rb})$ family of proteins and P53 and to include telomerase is some of the critical events that contribute to the development of malignancy [4].

\section{The virus components and physical properties as}

Papilloma viruses are small, non -enveloped, icosahedral DNA molecule of about 8000base pair (bp) that is bound to cellular histones and contain in a protein capsid composed of 72 pentameric capsomers. The capsid contains two structural proteins-Late (L1)(55KDa) which are both virally encoded. Virus like particles (VLPs) can be produced by the expression of L1, alone or in combination with L2, in mammalian or non $\mathrm{b}$-mammalian expression system.

The genome of all HPV types contains approximately eight (ORFs) that are all transcribed from a single DNA strand. The (ORF) can be divided in to three functional parts, early $(\mathrm{E})$ region that encodes proteins (E1---E7) necessary for viral replication.

The late $(\mathrm{L})$ region that $\mathrm{b}$ encode the structural protein (L1----L2) that are required for vision assembly, and a largely non-coding part that is referred to as the long control region (LCR), which contain elements that are necessary for the replication and transcription of viral DNA. The viral E proteins are transcribed from the early promoter [5].

The E1 and E2 proteins Of HPV act as factors that recognize origin of replication, E2 protein is also the main regulator of viral gene transcription. E4, despite its name is believed to be involved in the late stage of the life cycle of the virus and E5 may function during both early and late phase. The E6 and E7 proteins target a number of negative regulators of the cell cycle. Primarily P105Rb and P53 respectively. 
During the life cycle, the primarily, E6 and E7 facilitates stable maintenance of viral episomes and stimulate differentiating cells to reenter the $S$ phase. The L1 and L2 proteins assemble in capsomers, which form icosahedral capsids around the viral genome during the generation of progeny virion.

The life cycle is thought to be initiated by the infection of basal epithelial cells, presumably at site of injury. Although several potential receptors have been reported, it is UN clear which of them is of physiological importance. Basal cells comprise the proliferating cellular component of stratified epithelia, in which the viral genome established when a low copy number, nuclear plasmid and early genes are expressed preferentially although at low levels [6].

The ability of HPVs to establish their genome in basal cells relies up on the E1 [7], E2, E6, and in some cases E7 genes. Normally when basal cells undergo cell division, the daughter cells that loss contact with the basement membrane and migrates in to the supra basal compartment with draws from the cell cycle and initiates a program of terminal differentiation.

However in HPV-positive human keratinocytes and cervical epithelial cells, the supra basal cells fails to withdraw from the cell cycle and continue to support DNA synthesis and express markers for cell proliferation [8].

HPV16 E7 has been shown to be necessary and sufficient to include supra basal DNA synthesis [9]. In addition, the E5 oncoprotein contributes quantitatively to this property both in HPV16 and HPV31. Within supra basal compartment, cells support the amplification of the viral genome, expression of capsid genes and assembly of progeny virus.

In contact of HPV - associated cervical cancer, the viral life cycle is perturbed in two fundamental ways.

First the progressive histopathological changes that arise in the cervical epithelium include the loss of terminal differentiation. This inhibition of differentiation process leads to a cellular states that cannot support the full viral life cycle.

Second the circular viral DNA genome, which normally resides as a nuclear plasmid, often becomes integrated in to the host genome and thereby becomes disrupted and it, s replication defective.

Integration events that are found in cervical cancer lead to the selective expression of E6 and E7 proteins which are hallmark of cervical cancer.
Rationale

Cervical cancer is the major problem of modern medicine. Human papilloma virus types 16 and 18 can be considered as main causative agent of cervical cancer in women.

These viruses considered to be wide spread sexually transmitted pathogen that can affect the epithelium of transitional zone of cervix and these viruses responsible for more than $70 \%$ of total cases of cervical cancer.

\section{OBJECTIVES}

\section{General objective}

To determine HPV type 16and 18 infections among young married ladies aged $18-24$ years.

\section{Specific Objectives}

- $\quad$ To determine presence of HPV 16 or 18 DNA among young married women aged (18-24 years) using convent PCR technique.

- To correlate between age and presence of HPV 16 and 18 DNA.

- To associate presence of HPV 16 or 18 DNA with different factors (age, occupation, and sex partner).

\section{MATERIALS AND METHODS \\ Study design \\ Clinical based descriptive study}

\section{Study area and duration}

The study was conducted in River Nile state from September to November 2017.

\section{Study population}

Young married women aged 18-24 years unvaccinated against HPV 16 and 18.

\section{Selection criteria}

- Young married women aged 18-24 years and UN vaccinated against HPV 16 or 18 were included.

- Women with malignancy, smokers, family history of cancer, heart diseases or with chronic diseases were excluded from this study.

\section{Sample size}

Fifty females in age18-24 years were enrolled in this study. They had no vaccination against high risk HPV types 16 or 18. Isolated DNA from urine samples by using the G- spin Total DNA Extraction Kit method.

Then made convention PCR technique, then moved in Agarose gel electrophoresis to show band pattern of amplified DNA molecules.

\section{Ethical approval}

The study was approved by the University of Elzaeim Alazhari. 


\section{DNA extraction}

Epithelial cells which shed in urine samples were collected from targeted populations by using sterile urine containers, centrifuged to harvest deposit of urine that contain epithelial cells.

Then DNA extracted was performed using extraction Kit (intron Biotechnology co, Ltd- Korea)

\section{METHODOLOGY}

Pipetted $200 \mu \mathrm{l}$ of urine deposit into $1.5 \mathrm{ml}$ micro centrifuge tube

- Added $20 \mu \mathrm{l}$ of protinase $\mathrm{K}$ and $5 \mu \mathrm{l}$ of RNase A solution into sample tube and gently mixed.

- $\quad$ Added $200 \mu \mathrm{l}$ of buffer into upper sample tube and mixed thoroughly.

- Then made incubation for lysate at 56C temperature for 10 minutes.

- Briefly centrifuged the $1.5 \mathrm{ml}$ tube to remove drops from the inside of the rid

- Added $200 \mu \mathrm{L}$ of absolute ethanol into the lysate, and mixed well by gently inverting 5-6 time, then centrifuged the $1.5 \mathrm{ml}$ tube to remove drops from inside the lid.

- Applied the mixture to the spin column without wetting the rim, closed cap and centrifuged at $13,000 \mathrm{rpm}$ for 1 minute, then discarded the filtrate and placed the spin column in anew $2 \mathrm{ml}$ collection tube. Added $700 \mu \mathrm{L}$ of Buffer WA to the spin column without wetting the rim and centrifuged for 1 minute at 13,000 rpm, Then discarded the flowthrough and reused the collection tube.

- $\quad$ Added $700 \mu \mathrm{l}$ of Buffer WB to the spin column and b centrifuged for 1 minute at 13,000rpm, then discarded the flow-through and placed into anew 2 $\mathrm{ml}$ collection tube, then again centrifuged for 1 minute to dry the membrane.

- Placed the spin column into anew 1.5 tubes and added $50 \mu \mathrm{l}$ of Buffer CE directly onto the membrane, then incubated for 1 minute at room temperature then centrifuged for 1 minute at $13,000 \mathrm{rpm}$ to elute.

Then entire extracted DNA was stored at -20 temperatures until processed by Polymerase Chain Reaction.

\section{Polymerase Chain Reaction} by PCR.

Total cellular DNA (100ng| $\mid \mu 1)$ was amplified

HPV type (16 and 18) with specific primers were used for conventional multiplex PCR (Table 1)

Table-1: Sequences of Type -Specific PCR Primers Used in Study

\begin{tabular}{|l|l|l|}
\hline HPV -genotype & Amplification (bp) & Sequence $\left(\mathbf{5}^{-}-\mathbf{3}^{-}\right.$) \\
\hline 16 & 457 & CAC AGT TAT GCA CAG AGC TGC \\
\hline 18 & 322 & CAC TTC ACT GCA AGA CAT AGA \\
\hline
\end{tabular}

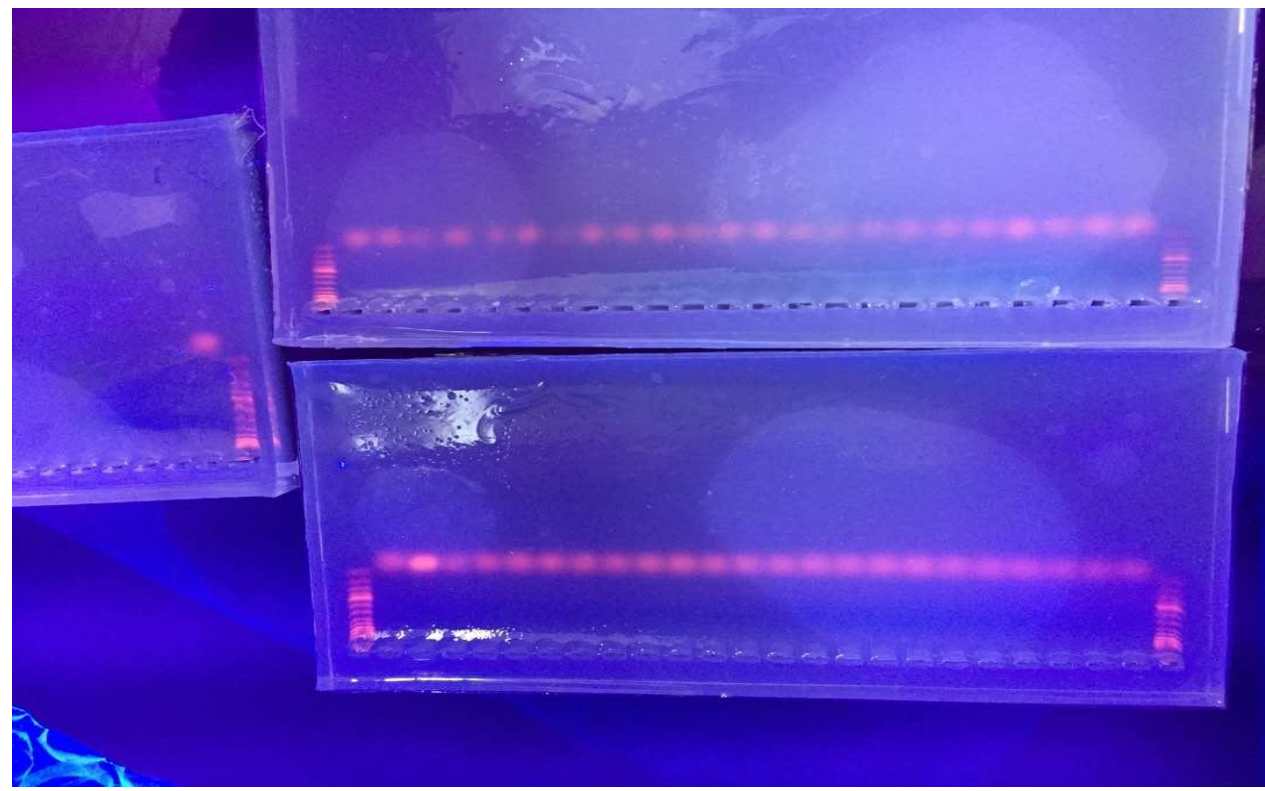

Fig-1: Pattern of 50 samples in gel electrophoresis

\section{Primers were prepared as}

1-Dissolved HPV -genotype 16 by $265 \mu$ distil water (Free RNA water) .

2- Dissolved HPV-genotype 18 by $429 \mu$ l distilled water.
Mixed well many times at room temperature (18-25C). Then centrifuged mixture by high speed centrifuged for 15 seconds to become ready for performance. 
These primers were designed to detect E7 and E6 open reading frame of HPV. Prepared on one tube, the ready master mix with primers, approximately 0.2 $\mathrm{mg}$ of extracted DNA was amplified in each dNTPs, $1 \mathrm{U}$ of Tag DNA polymerase, $2.5 \mu$ l of 10X PCR buffer.

20 Pmol of each primer, the reaction mixture was first heated at 94 temperature for 4 minutes and amplification was done for 30 cycle using PCR program .It was take one hour and 10 minutes .

The amplified products were resolved by electrophoresis on the $20 \%$ agarose gel. Which prepared as: Added 100 buffer (TBE) Trise Base (EDTA)) to $2 \mathrm{gm}$ Agar and heated at 60 temperature, before cooling I added ethidium bromide ( 2 micro) to each $100 \mathrm{ml}$.

Ethidium Bromide makes as visualizer), then prepared chamber of gel by made of 5 micro from Ladder on each edge of gel chamber. Then the running started from negative to positive direction about 22 minutes of a time. Then transferred stained gel with ethidium bromide to visualize on a UV Transilluminator.

\section{RESULT}

The overall urine specimens from all 50 married ladies in age of 18-24 years these were unvaccinated against HPV type 16 or 18 were not detected in a PCR process.

\section{DISCUSSION}

This study is the first cross-sectional studies with the aim to estimate the early impact of the HPV infection in young married ladies in River Nile state.

We assessed HPV infection in urine samples in 18-24 years old married ladies whom had not been offered the vaccine against HPV 16 or 18 which classified as high risk and had close relationship to cervical cancer.

The study was supported with positive control to evaluate PCR processing which was used in this study.
All 50 samples are negative for presence of any type of HPV 16 or 18, this indicates for behaviors which done by individuals to protect themselves against infection, these behaviors are:

\section{CONCLUSION}

HPV - related cervical disease is a significant burden .Persistent infection with an oncogenic HPV in the presence of co-factors is necessary to develop cervical cancer.

In all samples of study, there is no sexually active women with multi partners, so it decrease the infection with high risk HPV type 16 or 18 which closely related to cervical cancer.

\section{RECOMMENDATION}

All good behaviors which mentioned above not enough to prevent infection of high risk HPV infection Vaccine will be effective in blocking initial infection, and it is anticipated that it will prevent the development of HPV -induced malignancies.

Drugs which used to treat HPV infection has not been highly successful due to the complexities of the HPV life cycle and limited number of enzymatic activities identified for HPV proteins.

\section{REFERENCES}

1. Fehrman., \& Laimins. (2002). Classification of papilloma viruses.

2. Staoler., \& Broker, 1986, Schneider, 1987, Frattini, 1996, Oguch, HPV infection

3. Hubaert., \& Lainins.(2002). HPV infection, 2002

4. Jeanetal. (1995). Flores Human Papilloma Viruses

5. Florest. (2000). Human Papilloma Virus Infection.

6. Fehrman. (2002). Classification of papilloma viruses.

7. Trallier, H., Franco, E. 1. (2016). The epidemiology of genital human papilloma virus infection.

8. Villierfs, E.M., Fanquet, C. (2004). Broker classification of papilloma viruses -Virology, 234(17-27)

9. Kouts sky, L. (1997). Epidemiology of genital human papilloma virus infection, 3-8. 